\title{
Salud Mental en tiempos de pandemia: La pandemia paralela
}

\author{
Robles-Sánchez J.I. ${ }^{1}$
}

Sanid. mil. 2021; 77 (2): 69-72, ISSN: 1887-8571

El coronavirus (COVID-19), identificado en China a finales de 2019, con un alto potencial de contagio y rápido incremento de su incidencia mundial de forma exponencial, se fue expandiendo, adaptando y originando cepas diferentes. Su transmisión fue declarada por la $\mathrm{OMS}^{1}$, el 12 de marzo de 2020, pandemia mundial, advirtiendo que, a medida que la pandemia de coronavirus se propaga rápidamente por todo el mundo, existe un considerable temor, miedo y preocupación entre la población en general y entre ciertos grupos en particular, como los ancianos, los sanitarios y las personas con enfermedades previas. La información dudosa o incluso falsa sobre los factores relacionados con la transmisión del virus, el período de incubación, su alcance geográfico, el número de infectados y la tasa de mortalidad real ha generado inseguridad y temor en la población. La situación se vio agravada por la insuficiencia de las medidas de control y la falta de mecanismos terapéuticos eficaces ${ }^{2}$. Incertidumbres que tuvieron y tienen consecuencias en varios sectores, con implicaciones directas para la vida cotidiana y la salud mental de la población.

El miedo es, sin duda, un potente motivador y activador de las conductas de protección ante las posibles amenazas, pero desaparece a medida que las personas se adaptan a la situación. En una pandemia, el miedo aumenta los niveles de ansiedad y estrés en los individuos sanos e intensifica los síntomas de aquéllos con trastornos psiquiátricos preexistentes ${ }^{3}$.

Continuaba en su informe la $\mathrm{OMS}^{1}$ que el principal impacto psicológico, hasta aquél momento, había sido un alto porcentaje de estrés o ansiedad, y añadía que con la implementación de medidas como la cuarentena y su interferencia en las actividades y los hábitos diarios, con seguridad, se iban a aumentar los niveles soledad, depresión, consumo nocivo de alcohol y otros tóxicos y hasta el comportamiento autolesivo.

Estas hipótesis se basaban en el conocimiento adquirido de los resultados de las investigaciones realizadas en las anteriores epidemias por Ébola (EVE), SARS-CoV o MERS-CoV, que pusieron de manifiesto que tales situaciones causan un profundo malestar psicológico y un amplio espectro de impactos psicosociales negativos. Desde reacciones psicológicas de miedo a contraer el virus y caer enfermo, tener miedo a morir, el sufrimiento por estar infectado, la separación de familiares y amigos, al estigma asociado a la enfermedad, así como sensación de impotencia. Esas emociones negativas tienden a intensificarse con las restricciones tomadas por las autoridades para dificultar la propagación del virus, como han sido el cierre de escuelas y negocios ${ }^{4}$.

\footnotetext{
1. Teniente Coronel Psicólogo. Retirado. Adscrito a la Inspección General de Sanidad de la Defensa. Dirección para correspondencia: jrobsan@oc.mde.es

Recibido: 16 de junio de 2021

Aceptado: 29 de junio de 2021
}

doi: 10.4321/S1887-85712021000200001
En el caso de la COVID-19, las intervenciones no farmacológicas, aunque esenciales para detener la transmisión del virus (el aislamiento domiciliario con la reducción de la movilidad, la clausura de colegios, los cierres de negocios, el teletrabajo, etc.), han provocado alteraciones psicológicas. A estos efectos psicológicos producidos por la enfermedad y por las medidas tomadas por las autoridades, sanitarias y/o políticas, para tratar de controlar la transmisión del virus, es a lo que algunos autores han denominado la "pandemia paralela5". De hecho "los efectos indirectos del COVID-19 pueden ser mayores que el número de muertes debidas al virus en sí", sentenció el Director General de la Organización Mundial de la Salud, Dr. Tedros Adhanom Ghebreyesus, en la sesión de apertura de la conferencia de prensa sobre la COVID-19 el 12 de junio de 2020. Durante las epidemias, la cantidad de personas cuya salud mental se ve afectada tiende a ser mayor que la cantidad de personas afectadas por la infección ${ }^{6}$, demostrando que las implicaciones para la salud mental pueden durar más y tener mayor prevalencia que la propia epidemia y que las derivadas psicosociales y económicas pueden ser incalculables si consideramos su resonancia en los diferentes contextos. Si hacemos caso a los expertos, esa "pandemia paralela" ha generado problemas de salud mental que se sentirán incluso mucho después de doblegar al coronavirus. La comisionada de salud de la UE, Stella Kyriakides, ha señalado que "un tsunami de necesidades de salud mental se avecina cuando la COVID-19 retroceda".

El 5 de octubre de 2020, ante la proximidad del Día Mundial de la Salud Mental, la $\mathrm{OMS}^{7}$ hizo públicos los resultados de una encuesta sobre el impacto del COVID-19 en los servicios de Salud Mental, Neurología y problemas por uso de sustancias en 130 de sus estados miembros. El informe mostró los graves efectos que estaban teniendo la pandemia sobre la salud mental y el bienestar de todos los países estudiados, advirtiendo de la baja capacidad de respuesta para afrontar la crisis que se avecinaba. El estudio mostró que la mayoría de los países experimentaban algunas interrupciones en los servicios de salud mental, con el mayor impacto en los servicios comunitarios y los servicios de prevención y promoción de la salud mental. Las razones de la interrupción incluyeron el número insuficiente y/o la redistribución del personal sanitario en respuesta a la COVID-19 (en el $30 \%$ de los países), el uso de instalaciones de salud mental como instalaciones de tratamiento o cuarentena COVID-19 (en el 19\% de los países) y el suministro insuficiente de equipos de protección personal (en el $28 \%$ de los países). Aunque 116 países $(89 \%)$ informaron que la salud mental y el apoyo psicológico eran parte de sus planes nacionales de respuesta a la pandemia, solo el $17 \%$ informó que había comprometido fondos adicionales con este fin. El duelo, el aislamiento, la pérdida de ingresos y el miedo están desencadenando problemas de salud mental o agravando los existentes. Con una capacidad de respuesta en apariencia 


\section{EDITORIAL}

baja, no está claro cómo el mundo afrontará este problema de salud mental que se avecina. La pandemia de COVID-19 ha interrumpido o detenido los servicios críticos de salud mental en el 93\% de los países de todo el mundo, mientras que su demanda está aumentando. Años de escasas inversiones en salud mental, especialmente en países de ingresos bajos y medios, nos han dejado vulnerables.

Para paliar e intentar superar las interrupciones de los servicios asistenciales cara a cara, un alto porcentaje de países $(70 \%)$ adoptaron la telemedicina o la teleterapia; sin embargo, existen importantes disparidades en la aceptación de estas intervenciones terapéuticas. Más del $80 \%$ de los países de ingresos altos informaron haber implementado la telemedicina y la teleterapia para cerrar las brechas en salud mental, en comparación con menos del $50 \%$ de los países de ingresos bajos.

La pandemia ha dado ocasión para que los estudiosos de los diferentes países investiguen sobre estos problemas psicológicos, con factores de riesgo muy similares en todos ellos a pesar de las diferentes idiosincrasias. Se cuentan por cientos las entradas en la web of science al buscar las publicaciones sobre los efectos de la pandemia en la salud mental, tanto sobre la población general como sobre los sanitarios en particular, pero si se acota la búsqueda limitándola a revisiones sistemáticas y/o meta análisis el resultado, como es obvio, disminuye de forma considerable. Las metodologías de las investigaciones difieren de forma considerable entre ellas: población objeto de estudio, instrumentos de medida aplicados, ventana temporal de la recogida de datos, etc. De hecho se encuentran gran variabilidad en las prevalencias $^{8}$ de los problemas psíquicos detectados, analizando los datos de China, España, Italia, Irán, Estados Unidos, Turquía, Nepal y Dinamarca. Los síntomas de ansiedad $(6,33 \%$ al $50,9 \%)$, depresión ( $14,6 \%$ al $48,3 \%)$, estrés postraumático $(7 \%$ al $53,8 \%)$, malestar psicológico $(34,43 \%$ al $38 \%)$ o estrés $(8,1 \%$ al $81,9 \%)$. Sin embargo, son comunes para todos ellos los factores de riesgo: ser mujer, joven ( $\leq 40$ años de edad), presencia de enfermedades crónicas y enfermedades psíquicas, estar en paro, ser estudiante y exponerse frecuente a las noticias concernientes a la COVID-19 en los diferentes medios de comunicación.

Por citar algunas de las investigaciones más cercanas a nuestro entorno, en España, un estudio ${ }^{9}$ realizado durante las primeras etapas de la pandemia sobre una muestra de más de tres mil adultos recoge que, para la gran mayoría, había tenido un gran impacto en su vida diaria, incluyendo cambios en sus rutinas diarias y la cancelación de actividades importantes. Aproximadamente el 36\% de los encuestados informaron de un impacto psicológico de moderado a severo, el 25\% mostró niveles severos de ansiedad, el $41 \%$ informó de síntomas depresivos y el $41 \%$ se sintió estresado. Especialmente afectados se habían visto las mujeres, los jóvenes y aquéllos que perdieron su trabajo durante la crisis. La preocupación mayor de los encuestados era la probabilidad de sufrir una crisis económica derivada de la pandemia. Como factores positivos, se encontraron el estar satisfecho con la información recibida, la realización de actividades de ocio y la percepción de sentirse sano.

Otro interesante estudio longitudinal realizado igualmente en población española ${ }^{10}$ tras un año de convivencia con la COVID19, compara los resultados de las cuatro oleadas habidas hasta el momento. Un $40 \%$ de la muestra reveló haber tenido algún problema de salud mental desde el inicio de la pandemia, de los cuales el 23,4\% señala haber recibido algún tipo de tratamiento. En general, en la cuarta oleada se observa un aumento significativo en la sintomatología depresiva, de ansiedad, de estrés postraumático, de síntomas somáticos y en la aparición de un ataque de pánico, pareciendo indicar una situación de desgaste y de indefensión de la población española al enfrentarse a una cantidad elevada de sucesos estresantes durante un tiempo prolongado (12 meses), cuyo final es indefinido y difícil de controlar. El género femenino sigue mostrando ligera mayor sintomatología en casi todas las áreas (ansiedad, depresión, estrés postraumático y síntomas somáticos). Las personas más jóvenes (18-30 años) siguen mostrando una mayor sintomatología depresiva y de ansiedad en comparación con el resto de los grupos, excepto para el estrés postraumático y los síntomas somáticos donde no se encuentran diferencias.

En definitiva determinan que la población española está sufriendo un desgaste psicológico derivado de la vivencia de importantes sucesos vitales estresantes y estamos lejos aún de una recuperación psicológica total, ya que a los 12 meses la situación ha empeorado considerablemente y es previsiblemente que siga empeorando hasta que no se llegue a la inmunidad de rebaño y a la progresiva recuperación de la actividad económica.

En Francia, un estudio publicado en marzo de $2021^{11}$ muestra los efectos de los confinamientos sobre la salud mental. Una persona de cada siete ha presentado un síndrome depresivo como resultado del primer confinamiento durante la primavera de 2020, persistiendo la tendencia en la actualidad. Las mujeres y los jóvenes son los más afectados. Las alteraciones del sueño, la pérdida del interés por hacer cosas, el humor depresivo y las dificultades de concentración aumentaron de forma significativa durante el primer confinamiento. El estudio destaca, en particular, que "el hecho de encontrarse ante un comportamiento violento, agresivo o degradante por parte del cónyuge o pareja también estaba vinculado al síndrome depresivo". Según el estudio, más de una de cada cinco personas que vivían en un apartamento sin balcón desarrollaron síndrome depresivo. Además, haber estado encerrado fuera de casa, haber vivido en hogares hacinados o, por el contrario, haber vivido solo o solo con un hijo o hijos aumentaba el riesgo de depresión.

Otro estudio comparativo entre las poblaciones alemana e inglesa ${ }^{12}$, obtiene los resultados siguientes: E1 25\% de los que respondieron, tanto en Alemania como en el Reino Unido, informaron de un empeoramiento subjetivo de los síntomas psicológicos generales y el $20-50 \%$ de los respondientes a la encuesta alcanzaron los límites clínicos para los síntomas depresivos y distímicos, así como para la ansiedad. Destacan de forma específica la necesidad de implementar sistemas de intervención para apoyar a la mayor cantidad posible de personas en general.

En los primeros escenarios de la pandemia, sobre todo en algunos ámbitos políticos y medios de comunicación, se utilizaron metáforas militares/metáforas de guerra en esos tiempos de adversidad para conjurar los riesgos que se avecinaban, mostrando imágenes de valentía, abnegación y sacrificio. No es nuevo, pues ya se habían utilizado en otras ocasiones como fue en "la guerra contra las drogas" o "guerra de precios", por citar algunos ejemplos. Por lo tanto, no resultó sorprendente que en la pandemia por COVID-19 se utilizaran imágenes, 


\section{EDITORIAL}

metáforas y lenguaje tomados del ámbito militar, aunque no fuesen del agrado de algunos. Se identificó al coronavirus como "el enemigo" a combatir y se evocaron términos como "batalla y movilización" para enmarcar la respuesta puesta en marcha contra la pandemia ${ }^{13}$. Resulta de interés la descripción que hacen estos autores del "daño moral" que ha supuesto para muchos profesionales sanitarios, en las primeras etapas de la pandemia, al tener que afrontar la escasez de recursos, decidiendo a quién se le ponía ventilación mecánica y a quién no, o no estaban capacitados para enfrentar una enfermedad de la que desconocían su fisiopatología y cuál era el tratamiento eficaz o tener que acompañar a los pacientes en su último aliento, suponiendo para muchos de ellos un dilema ético que les abocó a un replanteamiento vital.

Sobre las Fuerzas Armadas y sus familiares encontramos algunos estudios. Uno de ellos ${ }^{14}$ expone como desde el inicio de la pandemia por COVID-19 en España, los miembros de la Fuerzas de Seguridad del Estado y de las Fuerzas Armadas, se movilizaron para garantizar la seguridad y movilidad del población y apoyar a las instituciones sanitarias, proporcionando personal para la atención, creando hospitales de campaña, traslado de enfermos y muertos, etc. El objetivo de este estudio fue determinar los niveles de burnout en estos profesionales. Los resultados muestran altos niveles de burnout $(28,5 \%)$ en todas las subescalas: agotamiento emocional $(53,8 \%)$, despersonalización $(58,0 \%)$ y falta de desarrollo personal $(46,3 \%)$.

Otro estudio focaliza su atención en la atención en salud mental para el personal militar de algunos países durante la pandemia $^{15}$. Reportan que, a medida que evolucionaba el brote de COVID-19, se han informado casos confirmados de COVID 19 entre el personal militar, a nivel mundial, incluidos casi 1.576 soldados en Rusia, más 4.000 en Estados Unidos, 1.500 soldados en Francia y 54 soldados entre las fuerzas de seguridad fronterizas de la India. Destacan que, si bien ha habido algunos informes sobre los desafíos sanitarios planteados por la COVID-19, ha habido pocos que prestasen atención a los aspectos únicos de la salud mental en las fuerzas armadas y el papel perturbador que juega el COVID-19 en esta población. El personal militar ha sido un grupo de trabajo muy importante en la lucha contra el COVID-19. Dado que el personal militar ya está en riesgo de problemas de salud mental (depresión, ansiedad, TEPT) durante épocas no pandémicas, el aumento de la carga de trabajo y el estrés que acompaña al COVID-19 puede aumentar la probabilidad de malestar psicológico. Un estudio investigó la salud psicológica de 642 personas que trabajaban en un hospital militar y encontró mayores tasas de detección de ansiedad. (29,44\%) y depresión $(36,45 \%)$ durante epidemia. Los factores de riesgo para padecer síntomas de ansiedad y/o depresión fueron las preocupaciones sobre estar infectados con COVID-19 ellos mismos o sus familias.

En nuestras Fuerzas Armadas, desde el inicio de la pandemia, el Mando se preocupó para que las medidas preventivas sobre la salud mental de nuestros hombres y mujeres se pusieran en práctica, activándose y coordinándose recursos desde la Unidad de Psicología de la Inspección General de Sanidad y desde el Servicio de Psiquiatría y Salud Mental del Hospital Central de la Defensa. Su trabajo ha quedado reflejado en sendos artículos publicados en el volumen 76(2) de esta revista.
La permanencia excesiva en una situación de restricciones impuestas está produciendo en la población la llamada "fatiga pandémica", definida por la OMS como una reacción natural y esperada a una adversidad sostenida y no resuelta en la vida de las personas. Se expresa como desmotivación para emprender conductas protectoras y buscar información, así como en sentimientos de complacencia, alienación y desesperanza. Puede resultar en la desmotivación para seguir las conductas protectoras recomendadas, emerge de forma gradual con el paso del tiempo y se ve afectada por una serie de emociones, experiencias y percepciones tanto individuales como colectivas. Es una situación que debe tenerse en cuenta por las autoridades competentes a la hora de dictaminar las medidas a adoptar y su duración en el tiempo, para evitar situaciones que pueden producirse por cansancio tanto físico como emocional.

Que las lecciones aprendidas de los diversos escenarios planteados por esta pandemia nos permitan estar siempre preparados y alertas para prevenir las amenazas futuras.

\section{REFERENCIAS:}

1. OMS, (2020). Recuperado de http://www.euro.who.int/fr/health-topics/noncommunicable-diseases/mental-health/data-and-resources/mental-healthand-covid-19

2. Ornell F, Schuch JB, Sordi AO, Kessler FHP. "Pandemic fear" and COVID-19: mental health burden and strategies. Braz J Psychiatry. 2020;42(3):232-235. doi: 10.1590/1516-4446-2020-0008

3. Shigemura J, Ursano RJ, Morganstein JC, Kurosawa M, Benedek DM. Public responses to the novel 2019 coronavirus (2019-nCoV) in Japan: mental health consequences and target populations Psychiatry Clin Neurosci. 2020;74:281-2.

4. Rodríguez-Rey R, Garrido-Hernansaiz H y Collado S (2020) Psychological Impact and Associated Factors During the Initial Stage of the Coronavirus (COVID-19) Pandemic Among the General Population in Spain. Front. Psychol. 11:1540. doi: 10.3389/fpsyg.2020.015407.- https://www.who.int/ publications/i/item/978924012455

5. Volkova, O.V. \& Shesternya, P.. (2021). Parallel pandemic: Psychological facilitation in medical assistance. Sibirskiy Psikhologicheskiy Zhurnal. 156-167. doi:10.17223/17267080/78/10.

6. Reardon S. Ebola's mental-health wounds linger in Africa. Nature. 2015;519:13-4.

7. WHO. The impact of COVID 19 on mental health, neurology and substance use services. The impact of COVID-19 on mental, neurological and substance use services (who.int)

8. Xiong J, Lipsitz O, Nasri F, Lui LMW, Gill H, Phan L, Chen-Li D, Iacobucci M, Ho R, Majeed A, McIntyre RS. Impact of COVID-19 pandemic on mental health in the general population: A systematic review. J Affect Disord. 2020 Dec 1;277:55-64. doi: 10.1016/j.jad.2020.08.001.

9. Rodríguez-Rey, R., Garrido-Hernansaiz, H., \& Collado, S. (2020). Psychological impact of COVID-19 in Spain: Early data report. Psychological Trauma: Theory, Research, Practice, and Policy, 12(5), 550-552. https://doi. org/10.1037/tra0000943

10. Ausín B., Castellanos M.A., González-Sannguino C., Muñoz M., Sáiz J., et al. Grupo 5.(2021). Conviviendo un año con la Covid-19. Estudio longitudinal de su impacto psicológico en la población española - Cátedra contra el estigma.

11. Direction de la Recherche, des Études, de l'Évaluation et des Statistiques. Confinement du printemps 2020 : une hausse des syndromes dépressifs, surtout chez les 15-24 ans Résultats issus de la 1re vague de l'enquête EpiCov et comparaison avec les enquêtes de santé européennes (EHIS) de 2014 et 2019. 2021:1185. https://drees.solidarites-sante.gouv.fr/sites/default/files/2021-03/ ER1185.pdf

12. Knolle F, Ronan L, Murray GK. The impact of the COVID-19 pandemic on mental health in the general population: a comparison between Germany 


\section{EDITORIAL}

and the UK. BMC Psychol. 2021 Apr 24;9(1):60. doi: 10.1186/s40359-02100565-y.

13. Jetly R., Vermetten E., Easterbrook B., Lanius R. y McKinnon M. (2020). Going to "War": Military Approach as the Antidote to Defeating COVID-19, Military Behavioral Health, 8:3, 243-247, DOI $10.1080 / 21635781.2020 .1765911$
14. Gómez-Galán J, Lázaro-Pérez C, Martínez-López JA, Fernández-Martínez MDM. Burnout in Spanish Security Forces during the COVID-19 Pandemic. Int J Environ Res Public Health. 2020 Nov 26;17(23):8790. doi: 10.3390/ ijerph 17238790

15. Guo X, Wu L, Yu X, Sun Z, Liu W. Mental Health Care for Military Personnel in the COVID-19 Epidemic. Mil Med. 2020 Sep 18;185(9-10):e1401e1405. doi: 10.1093/milmed/usaa127. 